\title{
Pancreas is an Unusual Initial Metastatic Site of Intracranial Hemangiopericytoma
}

\author{
Kamuran İBiş, ${ }^{1}$ Mert SAYNAK, ${ }^{2}$ Tulin YALTA, ${ }^{3}$ Cem İBiş, ${ }^{4}$ Zafer KOÇAK, ${ }^{2}$ Ahmet KARADENiZ ${ }^{1}$ \\ 'Department of Radiation Oncology, İstanbul University, Institute of Oncology, İstanbul-Turkey \\ ${ }^{2}$ Department of Radiation Oncology, Trakya University Faculty of Medicine, Edirne-Turkey \\ ${ }^{3}$ Department of Pathology, Trakya University Faculty of Medicine, Edirne-Turkey \\ ${ }^{4}$ Department of General Surgery, İstanbul University İstanbul Faculty of Medicine, İstanbul-Turkey
}

\begin{abstract}
SUMMARY
Intracranial hemangiopericytoma (HPC) is rare and aggressive tumor with local recurrences as well as distant metastases. The majority of metastases are encountered in bone, lung, and liver. Pancreatic metastasis is extremely rare. Described in the present report is the case of a 41-year-old woman who had undergone surgical resection of intracranial HPC 16 years earlier. The tumor recurred 3 years after the operation and was successfully managed with surgery followed by adjuvant radiotherapy. Thirteen years later, an isolated pancreatic metastasis developed. Patient underwent Whipple procedure for pancreatic head lesion and received adjuvant radiotherapy. Patient died of extensive disease in lungs, bones, mediastinum, cranium and peritoneal carcinomatosis in abdomen 17 months after pancreatic surgery.
\end{abstract}

Keywords: Hemangiopericytoma; metastases; pancreas; radiotherapy; surgery.

Copyright @ 2016, Turkish Society for Radiation Oncology

\section{Introduction}

Hemangiopericytoma (HPC) is a rare mesenchymal tumor originating from Zimmerman pericytes.[1] It can theoretically be seen anywhere in the human body where capillary bed is present. Soft tissue of lower extremity, pelvis, and retroperitoneum are areas where HPC is most often encountered. Intracranial HPC is rare, with a male predominance in fourth and fifth decades of life, and it is generally related to meningeal tissue.[1,2] Intracranial HPCs correspond to $2-4 \%$ of meningeal tumors and to less than $1 \%$ of all central nervous system (CNS) tumors. The most frequent areas of extraneural metastases are bones, lungs, and liver.[3] Pancreatic metastasis is extremely rare. Presently described is the second case reported in the literature with isolated pancreatic metastasis as the first distant metastatic site, which was followed-up with positron emission tomography-computed tomography (PETCT) imaging.

\section{Case Report}

A 41-year-old woman was admitted to general surgery with jaundice. She had no history of alcohol use or smoking. She had no comorbidities. Her father died due to lung cancer. She underwent surgical resection for intracranial HPC 16 years ago. Three years after surgery, a local recurrence developed that was managed with redo surgery followed by adjuvant radiotherapy (RT) (5 fractions of 2 Gy per week, total dose 56 Gy, 2D external RT with Cobalt-60).

Abdominal CT revealed $4 \times 5 \mathrm{~cm}$-sized solid mass in 

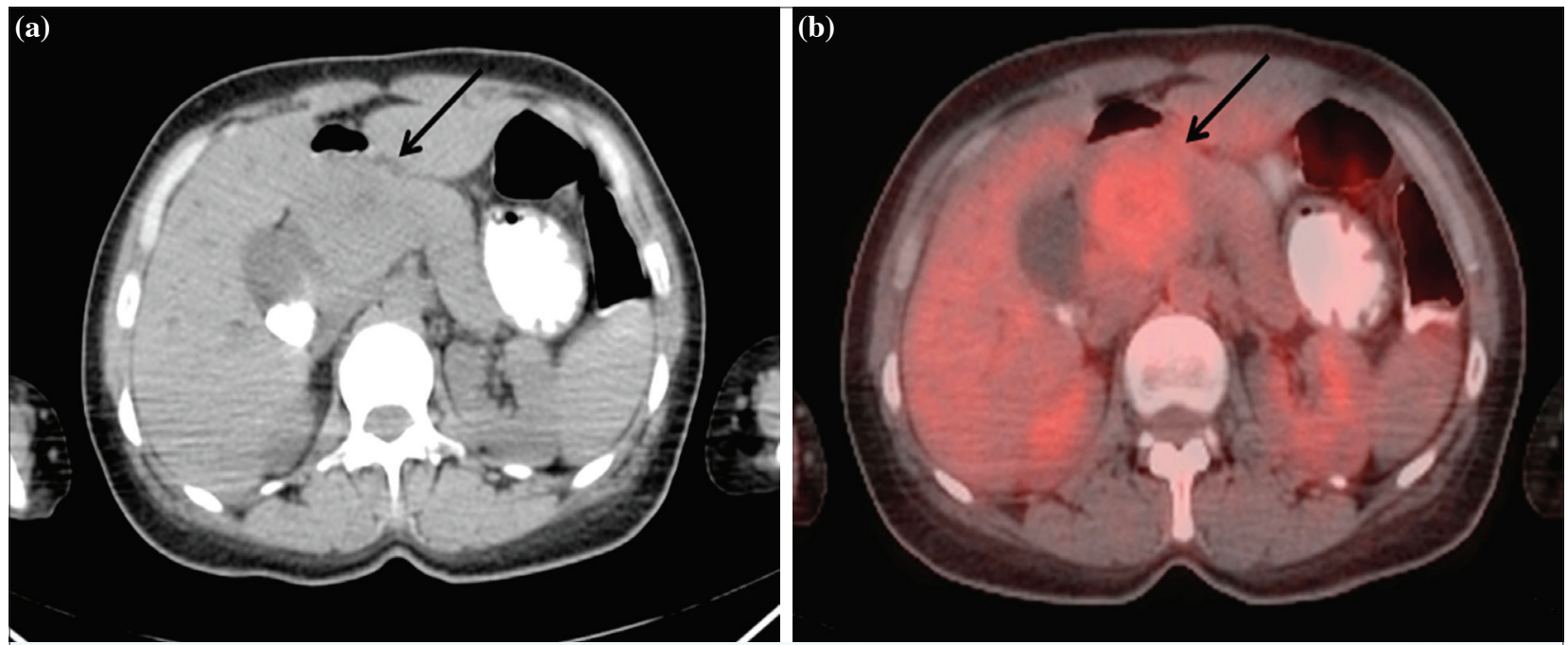

Fig. 1. (a) Axial CT demonstrates a soft-tissue mass (arrow) in the head of pancreas. (b) Axial F-18 FDG PET/CT fusion imaging demonstrates FDG uptake.

pancreatic head. Locally recurrent disease in cranium was excluded after cranial magnetic resonance imaging (MRI). F-18 fluorodeoxyglucose (18-FDG) PET/ CT imaging pointed to pancreatic head with slightly increased FDG uptake (SUVmax=4.2) in the mass (Figure 1a, b). There was no distant metastasis in PET/ CT images.

Patient underwent standard Whipple procedure with lymphadenectomy. Histopathological examination showed $6 \times 6 \times 4.5 \mathrm{~cm}$-sized malignant pancreatic HPC (Figure 2), with lymphovascular invasion. Mass was excised with negative surgical margins. Lymph node involvement was negative in 22 excised lymph nodes.

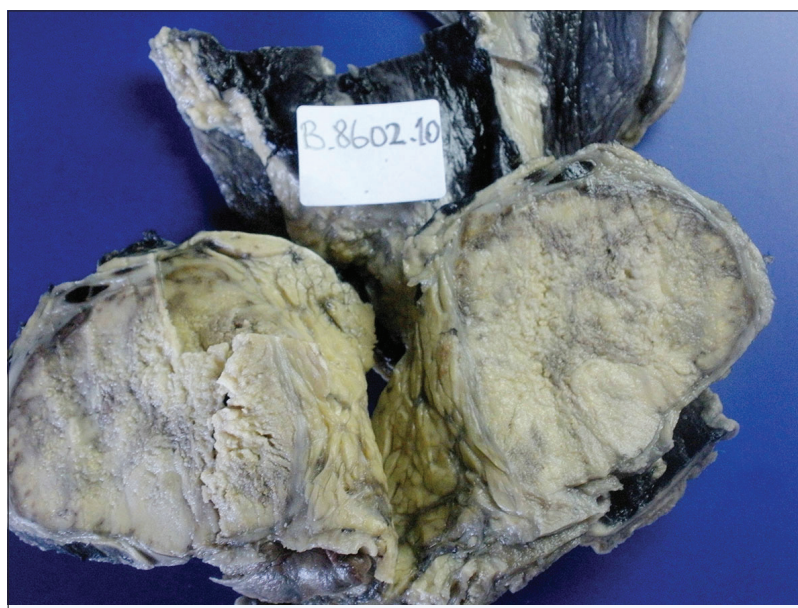

Fig. 2. Macroscopy demonstrating the $6 \times 6 \times 4.5 \mathrm{~cm}$-sized tumor in the head of the pancreas.
Previous cranial HPC pathology specimen (Figure 3a) was re-examined and compared with pancreatic

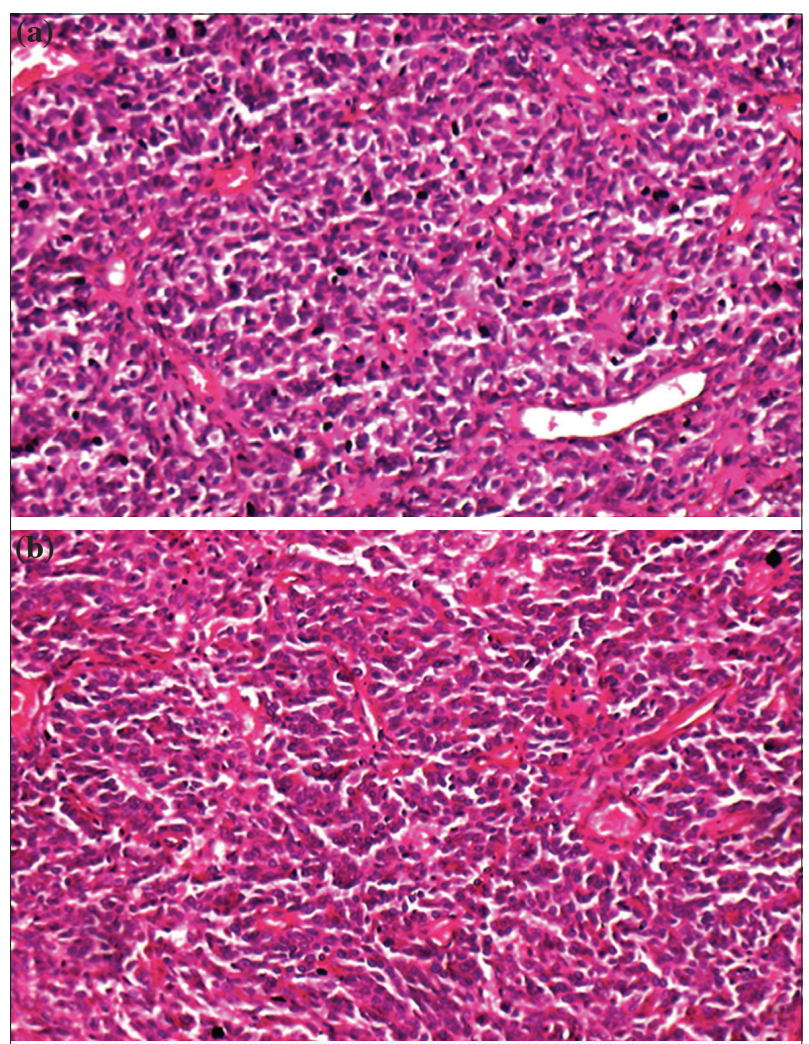

Fig. 3. Microscopy of tumors in the brain (a) and pancreas (b), respectively, typically demonstrating the spindle-shaped and tightly packed mesenchymal cells surrounding staghorn blood vessels (H\&E: hematoxylin and eosin, x100). 

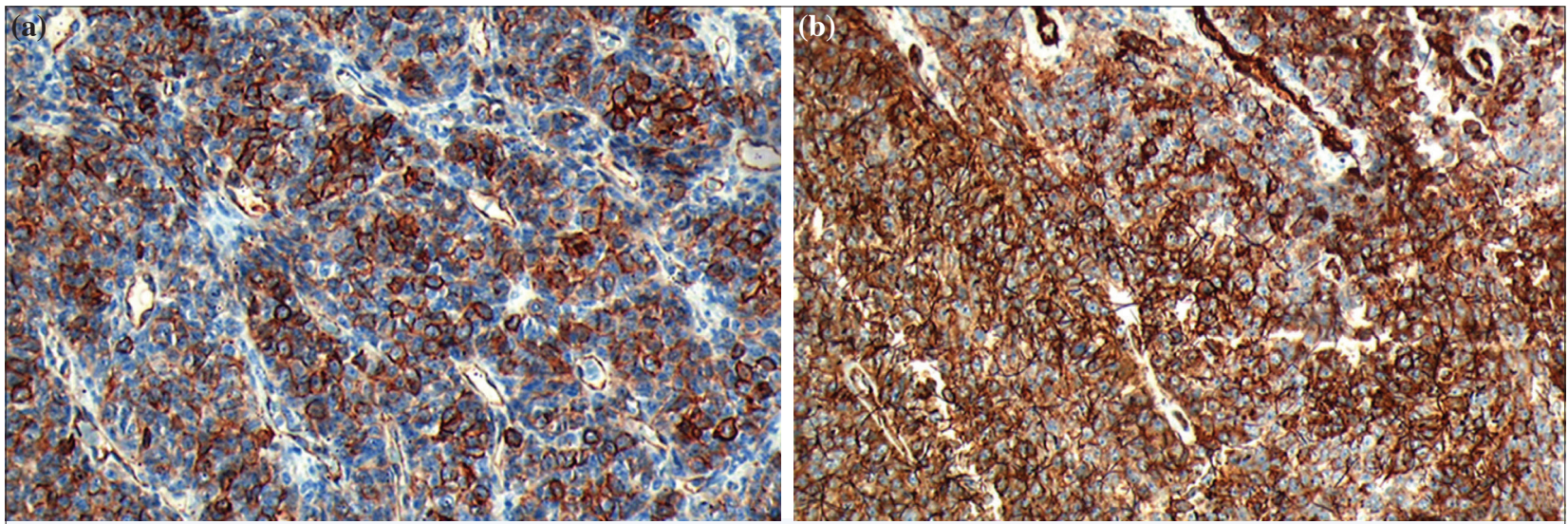

Fig. 4. Diffuse CD34 positivity of the spindle-shaped tumor cells in the brain (a) and pancreas (b), respectively (IHC: immunohistochemistry, x100).

specimen (Figure 3b). Tumors in brain and pancreas were found to be similar. Microscopy revealed mesenchymal tumor with increased number of branched blood vessels (staghorn vessels) surrounded by tightly packed nests of spindle-shaped cells similar to pericytes, which are found around blood vessels. The tumor cells were of little difference in size, with an elongated to pleomorphic nucleus, and appeared to be immunohistochemically positive for CD34 (Figure 4a, b) and vimentin, but negative for CD31. Ki-67 index was found to be around $10-15 \%$.

Pancreatic tumor was interpreted as a distant metastasis of the former meningeal HPC. Patient received adjuvant external RT (5 fractions of $180 \mathrm{cGy}$ per week, total dose 50.4 Gy, through 3D-CRT). Patient was admitted to hospital 17 months later with abdominal dis- tention. Abdominal sonography revealed diffuse ascites. CT, PET/CT (Figure 5) and cranial MRI showed extensive disease in lungs, bones, mediastinum, cranium and peritoneal carcinomatosis in abdomen. Patient died soon after admission.

\section{Discussion}

Intracranial HPCs demonstrate clinically and biologically aggressive behavior even after radical surgery followed by adjuvant RT. Local recurrences (up to 90\%) and extracranial metastases (up to 33\%) are frequent. [4-6] The most frequent areas of extraneural metastases are bones, lungs, and liver.[3] Whereas preferred treatment for intracranial HPC is surgical resection, the chance for definite cure is very low with surgery
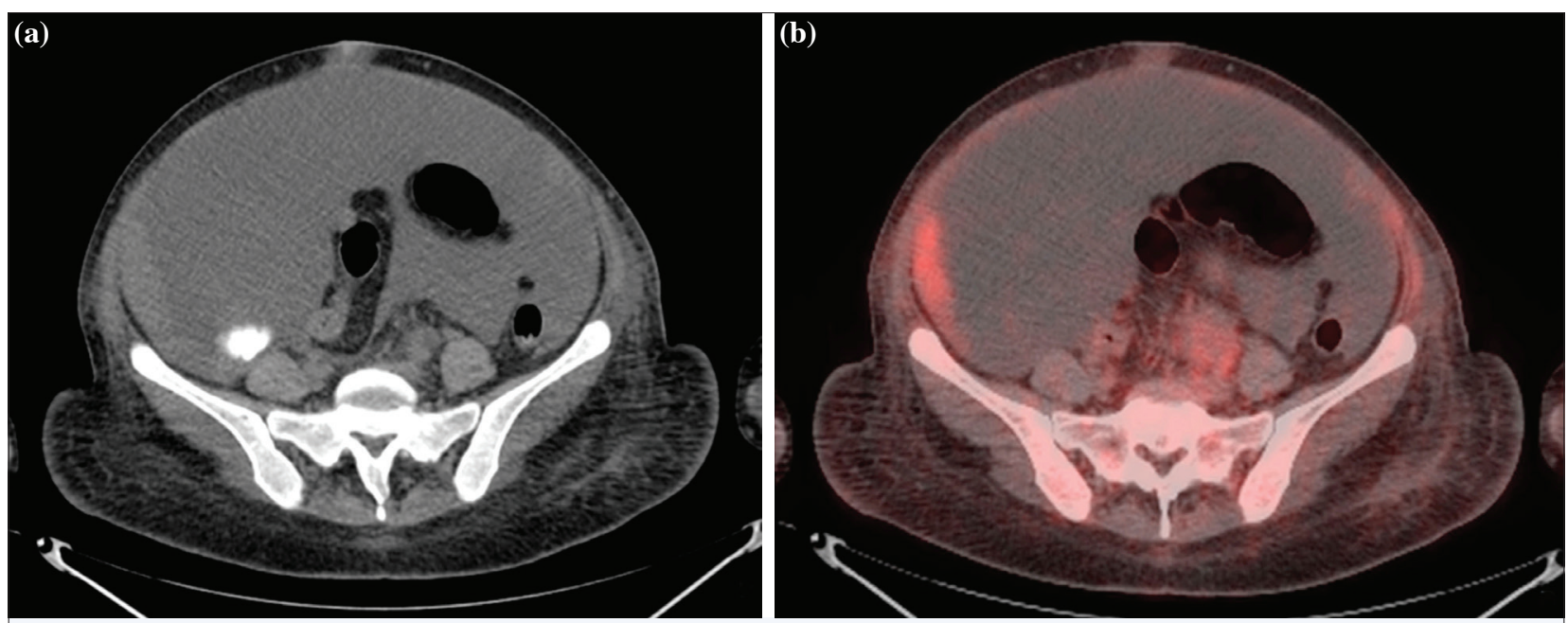

Fig. 5. (a) Axial CT demonstrates massive ascites and peritoneal implants. (b) Axial F-18 FDG PET/CT demonstrates massive ascites and high FDG uptake in peritoneal implants. 
alone. Intracranial HPC is known as a radiosensitive tumor. There is some research on role of adjuvant RT in treatment of recurrent intracranial HPCs.[7,8]

Hypervascular tumors are reported to be prone to bleed intraoperatively with high mortality rates (9-24\%) for intracranial HPCs.[9,10] Complete resection in the initial operation has been found to be highly correlated with long survival and late recurrence rates. Due to high risk of intracranial and extracranial metastases after surgical resection, it is suggested that RT be added to surgery in the treatment of intracranial HPCs. $[4,8]$ Guthrie et al.[3] reported progression-free survival as 74 months in irradiated, and 29 months in nonirradiated patients after surgery for HPCs. Doses of irradiation higher than 50 Gy have been recommended to avoid early recurrence and for local control after surgery.[10,11] Recently, Ghia et al.[12] demonstrated better local control in their study that included 63 intracranial HPC patients who received RT doses of more than 60 Gy following total resection of tumor. The most common metastatic sites are bone, liver, lungs, abdominal cavity, lymph nodes, skeletal muscle, kidneys, pancreas, skin, subcutaneous tissue, breast, adrenal glands, gallbladder, diaphragm, retroperitoneum, and heart.
We could find only 19 reports with 24 cases of pancreatic metastasis following primary intracranial HPC in English literature review (Table 1).[2,7,8,13-28] In only 3 cases was pancreas the first metastatic site, and concomitant local recurrences were reported in 2 out of 3 cases. [2,15,24] Iwaki et al.[15] irradiated their patient with isolated pancreatic metastasis 15 years after initial surgery. Trout et al.[2] presented a case of recurring meningeal HPC that led to additional resections 3 , 8 , and 12 years after primary resection. Eight months following the last resection, CT revealed a $6.3 \times 4.9 \mathrm{~cm}$ sized metastasis in pancreatic head. Standard Whipple procedure was performed. No information was provided about adjuvant therapy or survival. Patient with meningeal HPC presented by Tanabe at al.[24] had recurrence after 15 years with $7 \times 15 \mathrm{~cm}$-sized metastasis in pancreatic head. Patient underwent pancreatic surgery and was re-evaluated due to frequent postprandial vomiting following the pancreatic surgery. Head CT imaging revealed intracranial local recurrence. RT for the brain tumor with concomitant chemotherapy was planned; however, therapy could not be completed because of development of visual disturbance and Gerstman syndrome following the combination therapy.

\begin{tabular}{|c|c|c|c|c|}
\hline Author & Age & Sex & $\begin{array}{l}\text { Sites of metastases (at the time of } \\
\text { diagnosis of pancreas metastases } \\
\text { or previous metastases) }\end{array}$ & $\begin{array}{l}\text { Concomitant local } \\
\text { recurrence }\end{array}$ \\
\hline Meredith[20] & 44 & $\mathrm{~F}$ & Bone, (liver, lung, mediastinum, pancreas autopsy) & Yes \\
\hline Pitkethly[22] & ND & ND & Pancreas ( 4 cases) & ND \\
\hline Kepes[17] & 40 & M & Lung, kidney, liver, pancreas & 1 year ago \\
\hline Akagi[13] & 42 & $\mathrm{~F}$ & Lung, liver, pancreas, vertebra & Yes \\
\hline Palacios[21] & 40 & M & Lung (pancreas, kidney autopsy) & Yes \\
\hline Jestico[16] & 47 & M & Kidney, lymph node, liver, pancreas & Yes \\
\hline Tanabe[24] & 65 & M & Pancreas & Yes \\
\hline Arita[14] & 57 & M & $\begin{array}{l}\text { Bone (pancreas, kidney, spinal cord } \\
\text { metastases at the autopsy) }\end{array}$ & $\begin{array}{l}4 \text { years ago multiple } \\
\text { intracranial masses }\end{array}$ \\
\hline Iwaki[15] & 40 & M & Lung, liver, bone, lymph node & ND \\
\hline Iwaki[15] & 64 & $M$ & Pancreas & No \\
\hline Mena[19] & ND & ND & 2 cases pancreas & ND \\
\hline Koyama[18] & 55 & M & Liver, lung, bone, pancreas & 3 years ago \\
\hline Galanis[6] & ND & ND & Pancreas ( 1 case) & ND \\
\hline Niwa[25] & 55 & M & Liver, lung,vertebra, pancreas & ND \\
\hline Suziki[23] & 65 & M & Bone, pancreas & Yes \\
\hline Galambos[5] & 58 & M & Lung, pancreas & Last recurrence 5 year ago \\
\hline Hoshi[26] & 45 & $\mathrm{~F}$ & Bone, pancreas & ND \\
\hline Trout[2] & 67 & $\mathrm{~F}$ & Pancreas & Yes \\
\hline Hiraide[27] & 65 & M & Lung, kidney, pancreas & Last recurrence 2 year ago \\
\hline Ramos[28] & 52 & M & Pancreas, ND & ND \\
\hline Our case & 41 & $\mathrm{~F}$ & Pancreas & No, last recurrens 13 years ago \\
\hline
\end{tabular}


Patient died of renal insufficiency following pancreatic metastasis.

Hiraide et al.[27] reported on variable intervals (924 years) between initial diagnosis and manifestation of pancreatic metastasis. All 12 of their patients died in the first year after detection of pancreatic metastasis secondary to intracranial HPC.[27] The patient with meningeal HPC in our case report was diagnosed 16 years after initial diagnosis and surgery with solitary pancreatic metastasis and died 17 months after pancreatic resection.

Local recurrences as well as distant metastases frequently accompany intracranial HPC. This is an aggressive disease and lifelong follow-up after curative surgery and/or adjuvant radiotherapy should be taken into account. The possibility of late, distant metastasis of intracranial HPC, even in pancreas, without local recurrences should be kept in mind. PET/CT is an effective screening modality for metastasis. Pancreatic metastasis seems to be associated with poor survival rate.

Conflict of interest: None declared.

\section{References}

1. Teh BS, Lu HH, Jhala DN, Shahab I, Lynch GR. Pancreatic head mass from metastatic meningeal hemangiopericytoma. Sarcoma 2000;4(4):169-72.

2. Trout AT, Elsayes KM. Multidetector CT of pancreatic hemangiopericytoma. Cancer Imaging 2009;9:15-8.

3. Guthrie BL, Ebersold MJ, Scheithauer BW, Shaw EG. Meningeal hemangiopericytoma: histopathological features, treatment, and long-term follow-up of 44 cases. Neurosurgery 1989;25(4):514-22.

4. Kim JH, Jung HW, Kim YS, Kim CJ, Hwang SK, Paek $\mathrm{SH}$, et al. Meningeal hemangiopericytomas: longterm outcome and biological behavior. Surg Neurol 2003;59(1):47-54.

5. Galambos C, Cortese C, Janney C. Pathologic quiz case: a 58-year-old man with a pancreatic mass. Arch Pathol Lab Med 2003;127(2):105-6.

6. Galanis E, Buckner JC, Scheithauer BW, Kimmel DW, Schomberg PJ, Piepgras DG. Management of recurrent meningeal hemangiopericytoma. Cancer 1998;82(10):1915-20.

7. Hoffman HJ, Duffner PK. Extraneural metastases of central nervous system tumors. Cancer 1985;56(7 Suppl):1778-82.

8. Jäs̈keläinen J, Servo A, Haltia M, Wahlström T, Valtonen S. Intracranial hemangiopericytoma: radiology, surgery, radiotherapy, and outcome in 21 patients.
Surg Neurol 1985;23(3):227-36.

9. Chang SD, Sakamoto GT. The role of radiosurgery for hemangiopericytomas. Neurosurg Focus 2003;14(5):14.

10. Sheehan J, Kondziolka D, Flickinger J, Lunsford LD. Radiosurgery for treatment of recurrent intracranial hemangiopericytomas. Neurosurgery 2002;51(4):90511.

11. Dufour H, Métellus P, Fuentes S, Murracciole X, Régis J, Figarella-Branger D, et al. Meningeal hemangiopericytoma: a retrospective study of 21 patients with special review of postoperative external radiotherapy. Neurosurgery 2001;48(4):756-63.

12. Ghia AJ, Chang EL, Allen PK, Mahajan A, Penas-Prado $\mathrm{M}, \mathrm{McCutcheon}$ IE, et al. Intracranial hemangiopericytoma: patterns of failure and the role of radiation therapy. Neurosurgery 2013;73(4):624-31.

13. Akagi $T$, Iwata $K$, Utsunomiya $T$, Yoshimura $H$. An autopsy case of meningioma with extracranial remote metastases. Acta Pathol Jpn 1974;24(5):667-71.

14. Arita K, Uozumi T, Sakoda K, Okamoto H, Naito M. Hemangiopericytic meningioma with both intra- and extra-cranial metastases. Case report and review of the literature. [Article in Japanese] Neurol Med Chir (Tokyo) 1987;27(4):329-35. [Abstract]

15. Iwaki T, Fukui M, Takeshita I, Tsuneyoshi M, Tateishi J. Hemangiopericytoma of the meninges: a clinicopathologic and immunohistochemical study. Clin Neuropathol 1988;7(3):93-9.

16. Jestico JV, Lantos PL. Malignant meningioma with liver metastases and hypoglycaemia. A case report. Acta Neuropathol 1976;35(4):357-61.

17. Kepes JJ, MacGee EE, Vergara G, Sil R. A case report. Malignant meningioma with extensive pulmonary metastases. J Kans Med Soc 1971;72(7):312-6.

18. Koyama H, Harada A, Nakao A, Nonami T, Kurokawa T, Kaneko T, et al. Intracranial hemangiopericytoma with metastasis to the pancreas. Case report and literature review. J Clin Gastroenterol 1997;25(4):706-8.

19. Mena H, Ribas JL, Pezeshkpour GH, Cowan DN, Parisi JE. Hemangiopericytoma of the central nervous system: a review of 94 cases. Hum Pathol 1991;22(1):8491.

20. Meredith JM, Belter LF. Malignant meningioma: case report of a parasagittal meningioma of the right cerebral hemisphere with multiple extracranial metastases to the vertebrae, sacrum, ribs, clavicle, lungs, liver, left kidney, mediastinum and pancreas. South Med J 1959;52:1035-40.

21. Palacios E, Azar-Kia B. Malignant metastasizing angioblastic meningiomas. J Neurosurg 1975;42(2):185-8.

22. Pitkethly DT, Hardman JM, Kempe LG, Earle KM. 
Angioblastic meningiomas; clinicopathologic study of 81 cases. J Neurosurg 1970;32(5):539-44.

23. Suzuki H, Haga Y, Oguro K, Shinoda S, Masuzawa T, Kanai N. Intracranial hemangiopericytoma with extracranial metastasis occurring after 22 years. Neurol Med Chir (Tokyo) 2002;42(7):297-300.

24. Tanabe S, Soeda S, Mukai T, Oki S, Yun K, Miyahara S. A case report of pancreatic metastasis of an intracranial angioblastic meningioma (hemangiopericytoma) and a review of metastatic tumor to the pancreas. J Surg Oncol 1984;26(1):63-8.

25. Niwa M, Kobayashi T, Kuchiwaki H, Furuse M. A case of hemangiopericytoma with multiple extracranial metastasis: a case report. [Article in Japanese] No
Shinkei Geka 1998;26(3):241-5. [Abstract]

26. Hoshi M, Araki N, Naka N, Koizumi M, Hashimoto $\mathrm{N}$, Onishi $\mathrm{M}$, et al. Bone metastasis of intracranial meningeal hemangiopericytoma. Int J Clin Oncol 2005;10(3):208-13.

27. Hiraide T, Sakaguchi T, Shibasaki Y, Morita Y, Suzuki A, Inaba K, et al. Pancreatic metastases of cerebellar hemangiopericytoma occurring 24 years after initial presentation: report of a case. Surg Today 2014;44(3):558-63.

28. Ramos LR, Marques PP, Loureiro R, Brito MJ, de Freitas J. Pancreatic metastasis of a meningeal hemangiopericytoma: a rare cause of obstructive jaundice. Endoscopy 2014;46 Suppl 1 UCTN:E135-6. 